\section{Divergence évolutive \\ extrême \\ d'un gène \\ homéotique : \\ le cas bicoid}

\section{François Bonneton} gène essentiel peut changer de fonction au cours de l'évolution. La recherche d'homologues de bicoid a montré que ce gène était spécifique des mouches et absent chez les autres insectes. $\varepsilon$ n fait, il s'avère que bicoid est un gène homéotique Hox3 très dérivé (c'est-à-dire très éloigné de son gène ancestral). Au cours de l'évolution des insectes, le gène Hox3 ancestral a perdu sa fonction homéotique pour acquérir un nouveau rôle maternel et dans les annexes embryonnaires. Dans la lignée menant aux mouches, une duplication de ce nouveau gène a ensuite eu lieu, suivie d'une divergence aboutissant à la création des gènes bicoid et zerknüllt. L'analyse de l'évolution de bicoid, comme celle de nombreux autres gènes du développement, montre la nécessité d'élargir le choix des espèces modèles pour éviter les généralisations hâtives faites à partir d'un modèle particulier. <

La découverte des gènes du développement chez la drosophile a permis la recherche d'homologues chez d'autres organismes modèles et chez l'homme. Nombre de ces gènes contiennent en effet des domaines conservés permettant de synthétiser des sondes et des amorces utilisées dans des réactions de PCR (polymerase chain reaction) pour le clonage de gènes proches chez d'autres espèces. L'exemple le plus célèbre de cette approche est l'utilisation de l'homéoboîte, un domaine de fixation à l'ADN, pour cloner les gènes homéotiques (Hox) chez de nombreux animaux [1]. Aujourd'hui, la génétique du développement ne s'intéresse qu'à un très petit nombre d'espèces modèles:drosophile, nématode, poisson-zèbre et souris, pour ne citer que les principales. Pourtant, si la conservation de voies du dévelop-

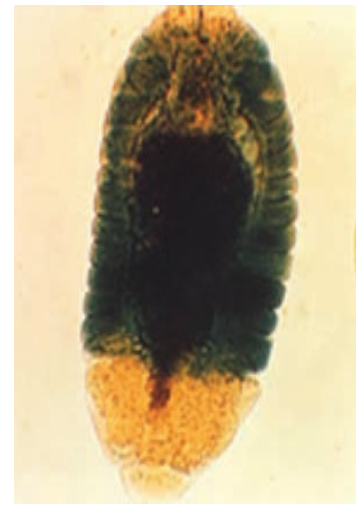

pement est souvent spectaculaire, il doit exister aussi de profondes divergences à l'origine même de la diversité morphologique des animaux. La génétique évolutive du développement («évo-dévo» pour les spécialistes) a justement pour objectif de comprendre les relations existant entre le développement et l'évolution [2]. Cette discipline récente a une approche expérimentale fondée sur les méthodes de la génétique du développement appliquées à des espèces choisies selon des critères évolutifs. Une des plus grandes surprises de l'évo-dévo naissante fut l'impossibilité de trouver le fameux morphogène bicoid ailleurs que chez les mouches. C'est pourtant l'analyse génétique de bicoid qui donna une réalité moléculaire au concept fondamental de morphogène, jusqu'alors purement théorique. Récemment, trois articles ont apporté un éclairage décisif sur cette énigme, dont la résolution montre comment un gène pourtant essentiel peut changer radicalement de fonction au cours de l'évolution [3-5].

\section{À la recherche de bicoid}

Chez la drosophile, bicoid joue un rôle essentiel dans la mise en place de l'axe antéropostérieur, comme l'in- 
dique l'absence de tête et de thorax chez les embryons mutants (Figure 1). Ce facteur de transcription à homéoboîte agit en établissant un gradient morphogénétique qui contrôle l'activation zygotique de différents gènes cibles dans la partie antérieure. Le morphogène nanos joue un rôle analogue dans la partie postérieure (Figure 1). Plusieurs équipes intéressées par l'évolution des gènes de segmentation tentèrent d'isoler bicoid chez des insectes au mode de développement différent de celui de la drosophile. Les résultats furent assez surprenants, puisqu'il s'avéra impossible de cloner bicoid en dehors du groupe des diptères cyclorrhaphes (de type mouche). En revanche, des gènes homologues de ceux qui codent pour les autres morphogènes précoces (nanos, hunchback et caudal) furent isolés chez plusieurs animaux. Par ailleurs, des transplantations de cytoplasme antérieur ${ }^{1}$ provenant de diverses espèces de mouches ne permettent pas toujours de restaurer le phénotype mutant bicoid de la drosophile [6], contrairement aux expériences similaires réalisées avec nanos [7]. Enfin, dans le complexe Hox de Drosophila melanogaster, entre Hox2 (proboscipedia) et Hox4 (deformed), se trouvent groupés trois gènes possédant un homéodomaine, mais n'ayant pas de fonction homéotique: bicoid, zerknüllt (zen) et z2 (Figure 2). Ces deux derniers gènes sont apparentés et jouent un rôle dans la mise en place de l'amnioséreuse, qui est l'annexe embryonnaire de la drosophile. II n'existe donc pas de gène Hox 3 typique chez cette espèce. L'ensemble de ces données suggéraient que bicoid, ainsi que zen et $z 2$, avaient connu une forte divergence de structure et de fonction au cours de l'évolution, et qu'ils pourraient dériver d'un gène Hox3 ancestral. Pour tester cette hypothèse, des homologues de bicoid et de zen furent recherchés chez d'autres insectes que la drosophile.

\section{Bicoid est un gène Hox3 très dérivé}

Chez tous les diptères cyclorrhaphes analysés, il fut possible d'isoler des gènes homologues de bicoid et de $z e n$, et de montrer que leurs profils d'expression étaient en grande partie conservés entre ces espèces $[8,9]$. La conservation fonctionnelle suggérée par ces résultats fut même testée pour bicoid, grâce à la technique d'interférence par l'ARN qui permet de produire des phénocopies $^{2}$ de type perte de fonction d'un gène par inacti-

${ }_{1}^{1}$ Technique qui consiste à prélever du cytoplasme de la partie antérieure d'un œuf d'une espèce et à l'injecter dans la partie antérieure d'un œuf d'une autre espèce, en l'occurrence des embryons mutants bicoid- de drosophile. Si le cytoplasme de la première espèce contient une activité de type bicoid, cette transplantation permet alors de restaurer le phénotype mutant bicoid de l'embryon de drosophile. Autrement dit, des embryons bicoid- transplantés parviennent à donner une larve qui éclot alors que cette mutation est normalement létale pour l'embryon.

2 Phénocopie: phénotype réversible d'une cellule ou d'un organisme imitant le phénotype résultant d'une modification génotypique. vation avec l'ARN double brin correspondant. Ainsi, que ce soit chez la mouche domestique (Musca) [8] ou bien chez un autre cyclorrhaphe issu d'une lignée plus basale (Megaselia) [10], I'interférence ARN permit d'obtenir des phénocopies ressemblant aux phénotypes mutants de bicoid décrits chez la drosophile. Par conséquent, la fonction de bicoid ne présente aucune divergence majeure parmi les diptères cyclorrhaphes.

Comme le montrent des résultats récents [5], la situation est très différente chez les diptères non cyclorrhaphes qui possèdent un homologue de zen, mais pas d'homologue de bicoid (Figure 3). L'analyse des séquences indique que zen et bicoid sont des gènes frères, et qu'ils dérivent probablement d'un gène Hox3 ancestral, confortant ainsi l'hypothèse émise auparavant $[9,11]$. Mais surtout, ce gène Hox3-zen des diptères non cyclorrhaphes est une sorte de chaînon manquant, puisque son expression combine des caractères des gènes zen et bicoid des diptères cyclor-

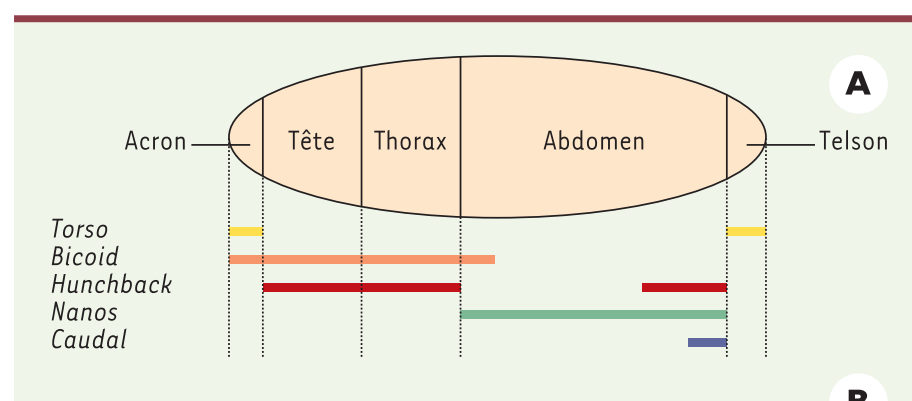

Concentration en protéine

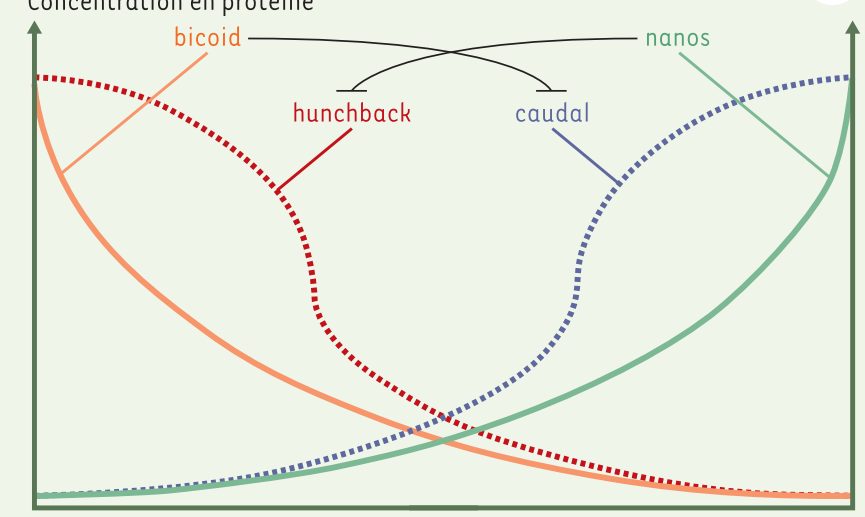

Antérieur

Figure 1. Les principaux déterminants maternels de la polarité antéropostérieur chez la drosophile. A. Schéma indiquant les régions de l'embryon affectées par la perte de l'un ou l'autre de ces morphogènes. Pour hunchback et caudal, le phénotype indiqué est celui obtenu en supprimant les deux phases d'expression, zygotique et maternelle. B. Diagramme représentant le taux d'expression des protéines maternelles le long de l'axe antéropostérieur de l'embryon. Les courbes terminées par une barre indiquent la répression de la traduction exercée par bicoid sur caudal et par nanos sur hunchback. 
rhaphes: expression maternelle (au cours de l'ovogenèse) comme bicoid, et expression dans les annexes embryonnaires comme zen (Figure 4). Dans la lignée menant aux diptères cyclorrhaphes actuels, il y aurait donc eu duplication d'un gène Hox3-zen ancestral, puis divergence des deux copies formées avec une perte de fonction pour chacune. Cette divergence se manifesterait ainsi par une séparation des tâches, la fonction maternelle étant dévolue à bicoid, alors que la fonction zygotique dans les annexes serait spécifique de zen. II est intéressant de noter que la duplication du gène

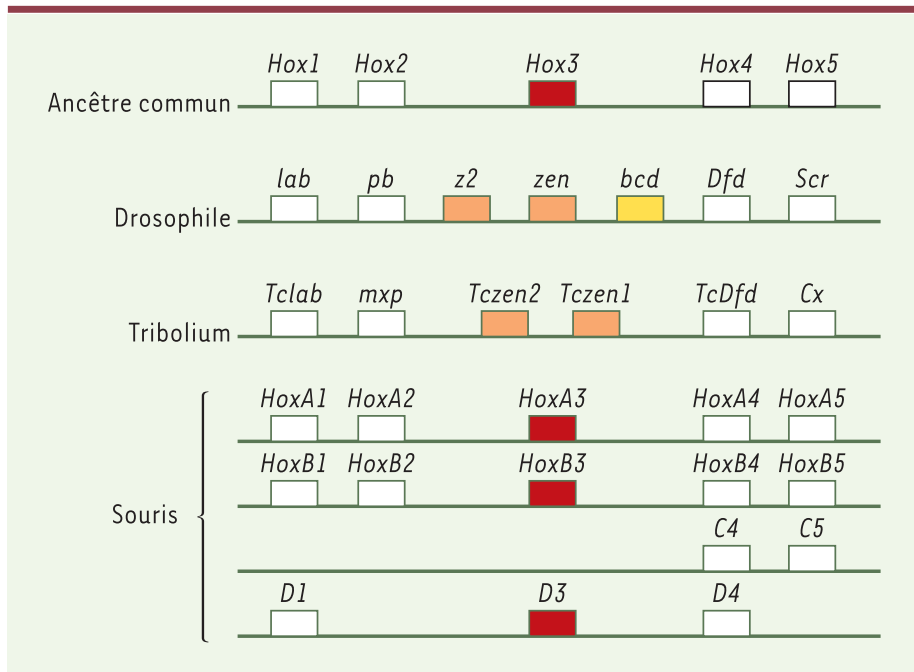

Figure 2. Structure du complexe Hox montrant les gènes du groupe antérieur chez la drosophile, le coléoptère Tribolium castaneum, la souris Mus musculus et chez leur ancêtre commun hypothétique. Les gènes de type Hox3 sont indiqués en rouge, et ceux de type zen sont en orange. lab: labial; $p b$ : proboscipedia; zen: zerknüllt; z2: zerknüllt 2; bcd (en jaune): bicoid; Dfd: Deformed; Scr: Sex combs reduced; Tc: Tribolium castaneum; mxp: maxillopedia; Cx: Cephalothorax (d'après [1] et [16]).

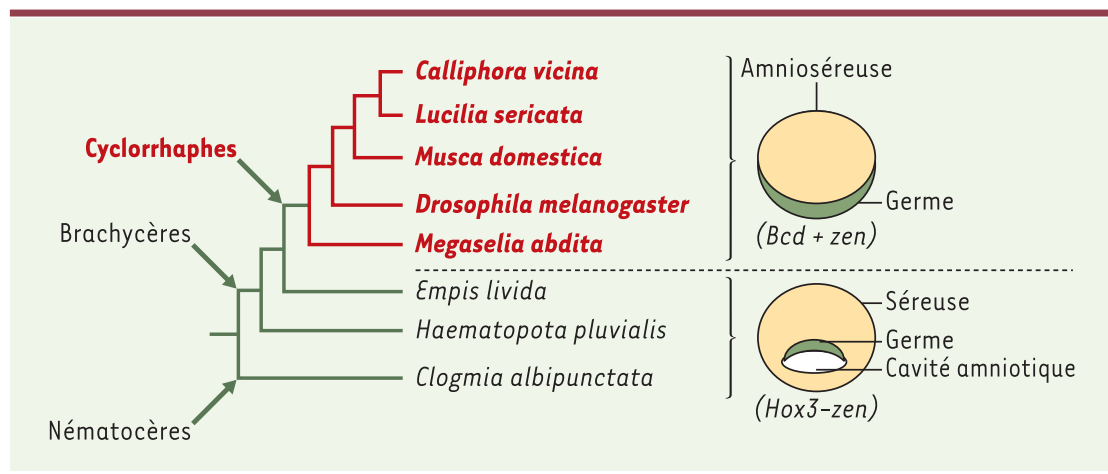

Figure 3. Phylogénie des diptères et type d'annexes embryonnaires. Les diptères cyclorrhaphes sont représentés en rouge. Ils possèdent les gènes bicoid et zen, ainsi qu'une annexe embryonnaire unique (amnioséreuse) située en position dorsale par rapport au germe embryonnaire. Les diptères issus de lignées plus basales n'ont pas de gène bicoid, mais un gène Hox3-zen, et deux annexes séparées (l'amnios et la séreuse) qui entourent le germe (d'après [5]). bicoid et la présence du gène zen (en deux copies chez cette espèce) dans le complexe Hox (Figure 2). L'impossibilité de cloner bicoid chez Tribolium était donc bien due à son absence, et non à sa divergence extrême. L'expression d'un des deux gènes Hox3-zen de Tribolium est restreinte aux annexes embryonnaires (Figure 4) [11]. Chez la sauterelle, le gène homologue présente une expression comparable à celle des diptères non cyclorrhaphes, c'est-à-dire à la fois dans les ovocytes et dans les annexes (Figure 4) [12]. Ces résultats indiquent qu'un gène Hox3-zen assurant une double fonction (maternelle et dans les annexes) est un caractère probablement partagé par tous les insectes autres que les diptères cyclorrhaphes (Figure 4).

\section{L'évolution de Hox 3 chez les arthropodes}

L'origine de bicoid étant connue, demeure le problème de la divergence initiale qui a transformé un gène homéotique Hox3 en gène impliqué dans la formation des annexes embryonnaires. En suivant la même démarche consistant à cloner et à étudier l'expression de gènes homologues dans des espèces de plus en plus éloignées des insectes, le travail de plusieurs équipes a permis récemment de résoudre ce problème. Les trois groupes proches des insectes sont les crustacés (le groupe frère des insectes), les myriapodes (mille-pattes) et les chélicérates (araignées, acariens) (Figure 4). Pour l'instant, les données existantes concernent quatre espèces de chélicérates [13-15] et une espèce de myriapode [4]. L'information majeure est que, dans tous ces organismes, l'expression embryonnaire du gène Hox3 est typique de celle d'un gène homéotique. Selon les espèces, deux à cinq segments antérieurs sont concernés. On n'observe pas d'expression maternelle, ni dans les annexes embryonnaires. Par ailleurs, les gènes 
homologues clonés chez un insecte primitif et chez divers crustacés ont une séquence plus proche de celle des Hox3 de type homéotique que de celle des Hox3 de type zen [16], mais leur expression n'est pas encore connue. Par conséquent, la divergence de Hox3 accompagnée d'une perte de sa fonction homéotique ne concerne pas tous les arthropodes et semble spécifique de la lignée des insectes les plus récents (Figure 4). Le fait que ces insectes puissent se passer d'un des cinq gènes homéotiques contrôlant l'identité des segments de la tête chez les autres arthropodes est un point intéressant qui n'a pas encore été étudié.

\section{Comment vivre sans bicoid?}

Est-il possible d'identifier le ou les gènes qui remplaceraient fonctionnellement bicoid chez les espèces dépourvues de ce gène? Les deux autres déterminants antérieurs précoces de la drosophile, Torso et hunchback (Figure 1), sont des candidats potentiels pour constituer l'organisateur antérieur primitif des insectes.

Torso et bicoid agissent sur des gènes cibles communs dans la partie antérieure terminale non segmentée de l'embryon (futur acron). En augmentant le nombre de copies de bicoid, il est possible de sauver le phénotype mutant torso [17]. Ces résultats montrent que torso et bicoid ont des fonctions qui sont en partie redondantes. Concernant hunchback, la protéine est répartie selon un gradient maternel antérieur indépendant de bicoid. Comme cette expression ressemble beaucoup à celle de bicoid, et qu'elle est conservée chez les insectes, il était tentant de supposer que la protéine constituait le morphogène antérieur primitif. Mais hunchback connaît une deuxième phase d'expression précoce qui est directement induite par bicoid, via un promoteur zygotique différent de celui qui règle l'expression maternelle. Pour étudier uniquement la fonction du gradient maternel de hunchback, il est possible de créer des lignées transgéniques dans lesquelles l'expression de ce gène est sous le contrôle exclusif du promoteur maternel, et donc indépendante de bicoid. En jouant sur le nombre de copies de ce transgène à expression maternelle, on observe un sauvetage du phénotype

${ }^{1} R$. Schröder vient de montrer [22] que les gènes orthodenticle et hunchback agissent ensemble pour assurer la détermination précoce antérieure chez le coléoptère Tribolium castaneum. Ils remplacent ainsi fonctionnellement le gène bicoid dont est dépourvu cet insecte. zygotique de hunchback, c'est-à-dire la formation de la tête et du thorax [18]. Par conséquent, les segments antérieurs peuvent se former uniquement grâce à hunchback maternel et indépendamment de toute activation par bicoid. En résumé, le rôle de bicoid dans la formation de la partie antérieure de l'embryon peut être en grande partie assuré par torso et par hunchback.

Cette redondance suggère que, au cours de l'évolution des diptères cyclorrhaphes, le nouveau gène bicoid se serait intégré dans un réseau de régulation préexistant qui aurait compris hunchback et torso. Puis bicoid aurait pris le dessus, par acquisition rapide de nouveaux gènes cibles dans ce réseau, dont le mieux connu est hunchback lui-même [19]. II est également possible d'envisager que bicoid ait remplacé un facteur ancestral qui reste à découvrir. Ce pourrait être un gène de type orthodenticle, puisque ce facteur possède un homéodomaine ayant la même spécificité de fixation que bicoid, et qu'il intervient dans le développement de la tête chez tous les animaux ${ }^{1}$.

\section{Conclusions}

L'histoire évolutive de bicoid révèle plusieurs mécanismes fondamentaux de l'évolution des gènes de développement. En premier lieu, l'importance essentielle des modifications de la régulation de ces gènes. Par exemple, la perte de la fonction homéotique de Hox3 et l'acquisition d'un rôle dans les annexes chez les insectes ne peut s'expliquer, à l'origine, que par des

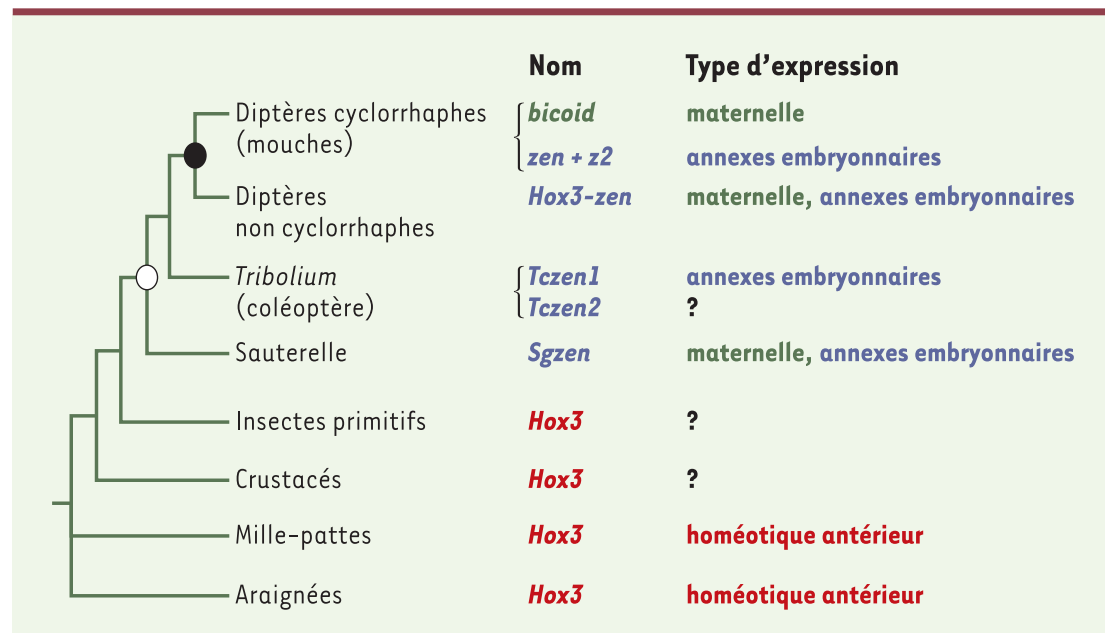

Figure 4. Évolution de Hox3 chez les arthropodes. L'ovale blanc symbolise la première transition évolutive, ou création du gène Hox3-zen, avec perte de la fonction homéotique de Hox3 et recrutement dans la formation des annexes chez les insectes «évolués». L'ovale noir symbolise la deuxième transition évolutive marquée par une duplication du gène Hox3-zen ancestral et divergence fonctionnelle des deux nouveaux gènes, zen et bicoid, chez les diptères cyclorrhaphes. Les points d'interrogation indiquent que l'expression n'a pas encore été étudiée. 
changements d'éléments régulateurs contrôlant l'expression spatiale et temporelle de ce gène. Mais les promoteurs ne sont pas les seuls à changer, les protéines et leurs partenaires subissent également des modifications importantes. Ainsi, la structure particulière de l'homéoboîte de bicoid (avec une lysine en position 50) et sa capacité exceptionnelle de fixer I'ARN (répression de la traduction de caudal) ont probablement joué un rôle fondamental dans l'évolution de cette protéine, en lui conférant une propriété que les autres homéoprotéines n'ont pas. Enfin, un autre mécanisme générateur de nouveauté, la duplication génique, a permis la création, chez les diptères cyclorrhaphes, des gènes zen et bicoid à partir d'un ancêtre de type Hox3-zen. Dans ce cas, la duplication a été suivie d'une divergence très importante à tous les niveaux, ce qui a abouti à une absence totale de redondance fonctionnelle entre les deux gènes chez la drosophile. Une divergence évolutive aussi importante n'est pas un cas exceptionnel. Par exemple, toujours au sein du complexe Hox, le gène fushi tarazu a également perdu sa fonction homéotique chez les insectes et les crustacés $[4,20,21]$. De manière plus générale, la plasticité des mécanismes embryonnaires semble être bien supérieure à ce que l'on pensait lorsque, lors de leur découverte, les premiers gènes du développement fascinaient surtout par leur conservation entre des espèces très différentes. Si cette plasticité nourrit les recherches de l'évolutionniste, elle révèle aussi clairement les limites d'une approche de la génétique du développement reposant sur un très petit nombre d'espèces modèles choisies selon des critères qui introduisent souvent un biais important au niveau des processus de l'ontogenèse (rapidité, canalisation, taille). D'où la nécessité de rechercher des espèces plus nombreuses et plus représentatives d'un groupe d'organismes avant de proposer des conclusions fondamentales. Heureusement, plusieurs techniques (RT-PCR, hybridation in situ, interférence ARN) permettent maintenant d'étudier assez facilement les gènes et leur fonction chez pratiquement toutes les espèces. L'introduction d'un peu d'évo-dévo dans un projet de biologie du développement présente au moins deux avantages. Premièrement, cela permet d'éviter le risque de travailler, sans le savoir, sur un système extrêmement dérivé et par là non généralisable. Enfin, en ouvrant une fenêtre sur la plasticité des mécanismes du développement, cette approche est capable de poser des questions essentielles liées notamment aux origines de la biodiversité [2]. L'exemple extrême de bicoid illustre à merveille les bonnes surprises que peut procurer l'évo-dévo. $\checkmark$

\section{REMERCIEMENTS}

Je remercie Vincent Laudet, Thomas Iwema et un arbitre anonyme pour la relecture critique de ce texte.

\section{SUMMARY}

Extreme divergence of a homeotic gene:

the bicoid case

Evolutionary developmental genetics (evo-devo) reveals that the plasticity of development is so important that every developmental biology project should carefully take this point into consideration. The example of bicoid, the first discovered morphogen, illustrates how an essential gene can change its function during evolution. The search for bicoid homologues showed that this gene is surprisingly specific to flies (cyclorraphan diptera) and absent in other insects. In fact, recent studies demonstrate that bicoid is a very derived Hox3 homeotic gene. During insect evolution, the ancestral Hox3 gene lost its homeotic function and acquired new roles in oocytes and embryonic annexes. Then, in the lineage leading to modern flies, a duplication of this new gene, followed by functional divergence, led to the formation of bicoid and zerknüllt. Both genes are located within the Drosophila Hox complex; however, they have no homeotic function. Thanks to the power of Drosophila genetics, it is possible to suggest that torso and hunchback may constitute the insect primitive anterior organizer. The bicoid evolutionary history reveals several fundamental mechanisms of the evolution of developmental genes, such as changes of gene regulation, modifications of protein sequences and gene duplication. It also shows the need for studying a wider range of model organisms before generalisations can be made from data obtained with one particular species. $\diamond$

\section{RÉFÉRENCES}

1. Deutsch J. Les gènes Hox et le rêve de Darwin. Med Sci 2000; 16: 205-11.

2. Laudet V. Vive la zoologie moléculaire! Med Sci 2002; 18: 234-6.

3. Brown SJ, Fellers JP, Shippy TD, et al. Sequence of the tribolium castaneum homeotic complex. The region corresponding to the Drosophila melanogaster Antennapedia complex. Genetics 2002; 160: 1067-74.
4. Hughes CL, Kaufman TC. Exploring the myriapod body plan: expression patterns of the ten Hox genes in a centipede. Development 2002; 129: 1225-38.

5. Stauber M, Prell A, Schmidt-0tt U. A single Hox3 gene with composite bicoid and zerknüllt expression characteristics in non-Cyclorrhaphan flies. Proc Natl Acad Sci USA 2002; 99: 274-9. 
6. Schröder R, Sander K. A comparison of transplantable bicoid activity and partial bicoid homeobox sequences in several Drosophila and blowfly species (Calliphoridae). Roux's Arch Dev Biol 1993; 203: 34-43.

7. Curtis D, Apfeld J, Lehmann R. nanos is an evolutionarily conserved organizer of anteriorposterior polarity. Development 1995; 121: 1899-910.

8. Shaw PJ, Salameh A, McGregor AP, Bala S, Dover $\mathrm{GA}$. Divergent structure and function of the bicoid gene in Muscoidea fly species. Evol Dev 2001; 3: 251-62.

9. Stauber $M$, Jackle $H$, Schmidt-0tt U. The anterior determinant bicoid of Drosophila is a derived Hox class 3 gene. Proc Natl Acad Sci USA 1999; 96 : 3786-9.
10. Stauber M, Taubert $H$, Schmidt-Ott U. Function of bicoid and hunchback homologs in the basal cyclorrhaphan fly Megaselia (Phoridae). Proc Natl Acad Sci USA 2000; 97 10844-9.

11. Falciani F, Hausdorf $B$, Schroder R, et al. Class 3 Hox genes in insects and the origin of zen. Proc Natl Acad Sci USA 1996; 93: 8479-84.

12. Dearden P, Grbic M, Falciani F, Akam M. Maternal expression and early zygotic regulation of the Hox3/zen gene in the grasshopper Schistocerca gregaria. Evol Dev 2000; 2: 261-70.

13. Damen WG, Tautz D. A Hox class 3 orthologue from the spider Cupiennius salei is expressed in a Hox-genelike fashion. Dev Genes Evol 1998; 208 : 586-90.
14. Telford MJ, Thomas RH. Of mites and zen: expression studies in a chelicerate arthropod confirm zen is a divergent Hox gene. Dev Genes Evol 1998; 208 : 59l-4.

15. Abzhanov A, Popadic A, Kaufman TC. Chelicerate Hox genes and the homology of arthropod segments. Evol Dev 1999; l: 77-89.

16. Cook CE, Smith ML, Telford MJ, Bastianello A, Akam M. Hox genes and the phylogeny of the arthropods. Curr Biol 2001; 11: 759-63.

17. Schaeffer V, Killian D, Desplan C, Wimmer EA. High bicoid levels render the terminal system dispensable for Drosophila head development. Development 2000; 127: 3993-9.

18. Wimmer $\varepsilon A$, Carleton $A$, Harjes P, Turner T, Desplan C. Bicoid-independent formation of thoracic segments in Drosophila. Science 2000; 287: 2476-9.
19. Bonneton F, Shaw PJ, Fazakerley C, Shi M, Dover GA. Comparison of bicoiddependent regulation of hunchback between Musca domestica and Drosophila melanogaster. Mech Dev 1997; 66: 143-56.

20. Hughes CL, Kaufman TC. Hox genes and the evolution of the arthropod body plan. Evol Dev 2002; 4: 459-99.

21. Mouchel-Vielh $\varepsilon$, Blin M, Rigolot C, Deutsch JS. Expression of a homologue of the fushi tarazu ( $f t z$ ) gene in a cirripede crustacean. Evol Dev 2002; 4: 76-85.

22. Schröder R. The genes orthodenticle and hunchback substitute for bicoid in the beetle Tribolium. Nature 2003; 422: 621-5.

TIRÉS À PART

F. Bonneton 